\title{
Lexical Group Unique Identifier
}

National Cancer Institute

\section{Source}

National Cancer Institute. Lexical Group Unique Identifier. NCI Thesaurus. Code C54177.

A unique identifier of a group of lexically equivalent atoms. For Eng lish language entries in the Metathesaurus, each string is linked to all of its lexical variants or minor variations by means of a common term identifier (LUI). In the Metathesaurus, therefore, an English "term" is the group of all strings that are lexical variants of each other. 